\title{
Über eine Eigenschaft der additiven Zerfällungen der Zahlen.
}

\section{Von S. Bergmann in Wien.}

Im Vorliegenden soll die Eigenschaft der Funktion $B(n)$, die gleich dem Unterschied der Anzahlen ist, welche anzeigen, wie oft die Zahl $n$ aus einer geraden und wie oft aus einer ungeraden Anzahl verschiedener der Zahlen $1,2,3 \ldots(p-2)$ additiv gebildet werden kann, untersucht werden. Wenn wir das Produkt

$$
f(x)=(x-1)\left(x^{2}-1\right)\left(x^{3}-1\right) \ldots\left(x^{p-2}-1\right)
$$

(wobei $p$ eine Primzahl bedeutet) nach Potenzen ron $x$ ordnen, ergibt sịch die Reihe

$$
n=\frac{(n-1)(p-2)}{\sum_{n=1}^{2}} B(n) x^{n}
$$

wo $B(n)$ die angegebene Bedeutung hat. Da es sich um eine Kongruenz-Eigenschaft handelt und unter $x$ in Folgendem stets ganze Zahl verstanden wird, so diurfen wir

setzen, somit

$$
x^{s(p-1)+k} \equiv x^{k}
$$

$$
\begin{aligned}
& f(x)=x^{p \cdots 1}\left\{1+B(p-1)+B(2 p-2)+\ldots B\left[\left.\frac{p-1}{2}(p-3)\right|_{\}}+\right.\right. \\
& +x^{n-2}\left\{B(p-2)+B(2 p-3)+\ldots+B\left[\frac{p-1}{2}(p-3)-1\right]\right\}+ \\
& +x\left\{B(1)+B(p)+B(2 p-1)+\ldots+B\left[\frac{1 \cdots \cdot 1}{2}(p-3)+1\right]\right\} .
\end{aligned}
$$

1) Der Modul $p$ der Kongruenz ist im folgemlen nicht melr besonders angegeben. 
Darin ist jeder Klammerausdruck gleich der Summe der Werte der Funktion $B$ für Argumente gleich den Gliedern der arithmetischen Progression

$$
\begin{aligned}
& s, s+p-1, \ldots s+\left(\frac{p-1}{2}\right)(p-3), \text { wenn } s \leqq \frac{p-1}{2}, \\
& s, s+p-1, \ldots s+(p-1)\left(\frac{p-5}{2}\right), \text { wenn } s>\frac{p-1}{2} .
\end{aligned}
$$

Anderseits besitzt $f(x)$ die Eigenschaft, daß sie kongruent 1 ist, wenn $x$ gleich einer Primitivwurzel von $p$ ist (da kein

$$
x^{*}=1
$$

ist, so verwandelt sich $f(x)$ in das Produkt 1.2.3..(p-2), welches nach Wilson schem Satz kongruent 1 ist). Ist $x$ gleich einer Nichtprimitivwurzel von $p$, so existiert mindestens ein $x$, bei welchen

und somit auch

$$
\left(x^{r}-1\right)=0
$$

$$
f(x) \equiv 0
$$

ist. Da für jede $f(x)$ die Beziehung gilt,

$$
\begin{gathered}
f(x) \equiv-\left[x^{p-1} \sum_{r=1}^{r=p-1} f(r)+x^{n-2} \sum_{r=1}^{r=p-1} r f(r)+\ldots\right. \\
\left.\ldots+x \sum_{r=1}^{r \cdots p-1} r^{p-2} f(r)\right]^{1)}
\end{gathered}
$$

so ist in unserem speziellen Falle

$$
f(x)=-\left[S_{0} x^{p-1}+S_{1} x^{n-2}+\ldots+S_{p-2} x\right],
$$

wobei unter $S_{k}$ die Summe der $k$ Potenzen aller Primitivwurzeln von $p$ gemeint wird. Ws ist nämlich nach dem Gesagten entweder $f(r) \equiv 1$ oder $f(r) \equiv 0$, je nachdem, ob $r$ eine Primitivwurzel oder Nichtprimitivwurzel ist, und somit ist also

$$
\sum_{r=1}^{r=1} r^{k} f(r) \equiv S_{k} .
$$

Ist $p-1=m=m^{\prime} P$ eine beliebige positive ganze $/$ ahl, $P$ das Produkt aus allen von einander verschiedenen in $m$ aufgehenden

1) Siebe über die „analytische Darstellung der Lösungen von Kongruenzen“. Monatshefte für Mathematik und Physik, XXVIII. Jahrg. 1917, S. 121. 
Primzahlen und $S_{k}$ die Summe der $k$ Potenzen aller Primitivwurzeln von $p$, so ist $S_{k}=0$, wenn $k$ nicht durch $m^{\prime}$ teilbar ist; ist aber

$$
k=m^{\prime} K
$$

ferner $Q$ der größte gemeinschaftliche Divisor von $K$ und $P(=Q R)$, und $r$ die Anzahl der in $R$ aufgehenden Primzahlen, so ist

$$
\left.S_{k} \equiv(-1)^{r} m^{\prime} \varphi(Q)^{1}\right)^{\prime}
$$

$\varphi$-Eulersche Funktion. Da schließlich die Koeffizienten bei gleichen Potenzen von $x$ in beiden Entwicklungen der $f(x)$ ubereinstimmen müssen, so ergibt sich, da6

$$
\begin{gathered}
B(p-s)+B(2 p-s-1)+\ldots+B\left[(p-s)+(p-1)\left(\frac{p-3}{2}\right)\right]= \\
\equiv-S_{s-1}=-(-1)^{r} m^{\prime} \varphi(Q) . \\
\text { Wenn } p-s \leqq \frac{p-1}{2} \text {, und } \\
B(p-s)+B[(p-s)+(p-1)]+\cdots+ \\
+B\left[(p-s)+(p-1)\left(\frac{p-5}{2}\right)\right] \equiv-S_{s-1} \equiv-(-1)^{r} m^{\prime} \varphi(Q),
\end{gathered}
$$

wenn

Beispiel:

$$
p-s>\frac{p-1}{2}
$$

$$
\begin{gathered}
1 \\
=7 \\
(x-1)\left(x^{2}-1\right)\left(x^{3}-1\right)\left(x^{4}-1\right)\left(x^{5}-1\right)=x^{15}-x^{14}-x^{13}+x^{10}+ \\
+x^{9}+x^{8}-x^{7}-x^{6}-x^{5}+x^{2}+x-1=-x^{5}+x^{4}+ \\
+2 x^{3}-x^{2}-x-2 \\
B(15)+B(9)+B(3)=-S_{3}=f[\text { gr. gem. Teiler }(6,3)=\varphi(3)=2 \\
15=1+2+3+4+5 \quad 9=2+3+4=1+3+5=4+5 \\
3=3=1+2
\end{gathered}
$$

Also zwei Zerfällungen in gerade und vier Zerfüllungen in ungerade Anzahl von Gliedern.

Da

Auberdem folgt noch aus dem Gesagten:

$$
[f(x)]^{k} \equiv f(x)
$$

ist, so düren wir, ohne das Resultat zu verändern, das Produkt

$$
f(x)=\prod_{n=1}^{n=p-2}\left(x^{n}-1\right)
$$

1) Dirichlet, Vorlesungen über Zahlentheorie, Supplement 7, 2. Aufl, S. 401 (FuBbemerkung). 
zu der $k^{\text {ten }}$ Potenz erheben, d. h.: wenn wir die Unterschiede der Anzahlen, welche anzeigen, wie oft die Zahl $n$ aus einer geraden und wie oft aus einer ungeraden Anzahl der Zahlen

$$
1,2,3, \ldots(p-2)
$$

additiv gebildet werden kann (wobei in jeder Zerfällung jede Zahl höchstens $k$ mal vorkommen darf), berechnen und deren Summe für die Werte $n==$

$$
s, s+(p-1), \ldots s+(p-1)\left[(k-1)\left(\frac{p-1}{2}\right)+\frac{p-5}{2}\right]
$$

resp. $s, s+(p-1), \ldots s+(p-1)\left[(k-1)\left(\frac{p-1}{2}\right)+\frac{p-3}{2}\right]$

bilden, so ist sie

$$
=-(-1)^{r} m^{\prime} ?(Q)
$$

Die erwähnte Zerfällung in die Zahlen der Reihe

$$
1,2,3 \ldots(p-2) \text {, }
$$

wo jede höchstens $k$-mal vorkommen kann, dürfen wir auch auffassen, als Zerfällungen in die Form

$$
s=1 x_{1}+2 x_{2}+3 x_{3}+\ldots+(p-2) x_{p-2},
$$

wo für $x=0$ oder eine beliebige Zahl, die $\leq k$ ist, genommen werden kann. Anstatt der früher angegebenen Differenz der Darstellungen mit geraden und ungeraden Anzahl der Glieder, mitssen wir den Unterschied der Anzahl der Zerfälluugen bilden, wo

$$
x_{1}+x_{2}+\ldots+x_{1-2}
$$

gerade oder ungerade Zahl ist.

Die Beziehung

$$
s=x_{1}+x_{2}+\ldots+x_{1} \quad(p-1)
$$

durfen wir auch in der Form

$$
a^{s}=a^{x_{1}} a^{x_{2}} \ldots a^{x_{q}} \quad(p) \quad(a \text { Primitivwurzel) }
$$

schreiben. Da alle

$$
x_{1}, x_{2} \ldots
$$

ein vollständiges Restsystem $(p-1)$ ausgenommen, modulo $(p-1)$ bilden; so bilden die Werte

$$
a^{x_{1}}, a^{x_{2}}, \ldots
$$

ein vollständiges Restsystem, die 1 von demselben ausgenommen, modulo $p$. Setzen wir $a^{s}=S$, so ist nach fruher Gesagtem die Summe der Differenzen der Darstellungen der Zahlen $S+$ diejenige, von $(p+S)+$ diejenige von $(S+2 p)+$ usw., als 
Produkt der geraden und ungeraden Anzahl voneinander verschiedenen Multiplikatoren der Reihe

$$
2,3,4 \ldots(p-1)
$$

kongruent $-(-1)^{r} m^{\prime} \varphi(Q)$, wo $Q$ der grölote gemeinschaftliche Teiler von $p$ und $k m^{\prime}, K=$ ind. $S_{k}$ ist. Die anderen Buchstaben behalten die alte Bedeutung: $p-1=m^{\prime} P . P$ das Produkt aus allen voneinander verschiedenen in $(v-1)$ aufgehenden Zahlen. Auf ganz ähnliche Weise läßt sich die Darstellung der Funktion

$$
\prod_{n=1}^{n=n-1}\left(x^{n}+1\right)
$$

in der Form eines Polynomes $(p-1)$ Grades durchfuhren und somit gewisse Schlitsse iiber die Anzahl der Zerfällungen der Zahl $n$ aufstellen. Da aber die Formeln für den allgemeinen Fall gewisse Komplikationen erleiden, soll im folgenden der Fall durchgeführt werden, bei welchem

$$
p^{\prime}-1=2 p_{1}
$$

ist, wo $\mu_{1}$ wieder eine Primzahl bedeutet. Wir werden den Fall in anderer Hinsicht verallgemeinern, indem wir anstatt des fritheren Produktes das Produkt

$$
g(x)=\coprod_{n=1}^{n=1}\left(x^{n-\cdots}-y\right)
$$

nehmen. Wir werden auch hier dic früher erwähnte Darstellung $g(x)=\cdots\left[x^{p-1} \sum_{r=1}^{r-p} g(r)+x^{p-2} \sum_{r=1}^{r-1} r g(r)+\ldots+x \sum_{r=1}^{r=p-1} r^{p-2} g(r)\right]$ anwenden. Um die

$$
\sum_{r=1}^{r=1--1}\left|r^{-s} g(r)\right|
$$

zu berechnen, müssen wir zuerst den Wert $g(x)$ für alle $x$ auffinden. Dazu setzen wir anstatt $x a^{\text {ind. } x}$, anstatt $y a^{\text {ind. } . y}$ ( $a$ eine Primitivwurzel von $p$ ) und erhalten dadurch:

Da $n$ alle Werte

$$
g(x)=\prod_{n=1}^{n=1^{\prime}-1}\left(a^{n \text { ind. } x}-u^{\text {ind. }, y}\right) .
$$

$$
1,2, \ldots p-1
$$

annimmt, so verschwindet $g(x)$, sobald die Gleichung

$$
n \text { ind. } x-\text { ind. } y \equiv 0 \quad(p-1) \quad(n \text { variable })
$$


eine Lösung besitzt. Das ist nicht der Fall, nur dann, wenn ind. $y$ durch den gröliten gemeinschaftlichen Teiler von $(p-1)$ und ind. $x$, nicht teilbar ist. Da $(p-1)=2 p_{1}$ ist, so kann der größte gemeinschaftliche Teiler entweder $\frac{p-1}{2}, p-1$ oder 2 sein. In beiden orsten Fällen ist

$$
g(x) \equiv \equiv\left(1-a^{2 \text { ind. } y}\right)^{\frac{p-1}{2}}
$$

resp.

$$
g(x)=\left(1-a^{\text {ind } y}\right)^{\prime \prime-1}
$$

weil

$$
a^{\frac{p-1}{2}} \equiv \pm 1, a^{\prime-1} \equiv 1
$$

i:t. Im letzten Falle verwandeln wir

$$
g(x)=\prod_{n=1}^{n=p-1}\left(a^{n \text { ind. } x}-a^{\text {ind. } y}\right)
$$

in

$$
\prod_{n=1}^{n=p-1}\left(a^{n \text { ind. } x-i n d . y}-1 .\right)
$$

Der Ausdruck

$$
a^{n \text { ind }, x-\text { ind, } y}
$$

(ind. $x$ gerade, ind, $y$ ungerade Zahl) ergibt für

$$
n=1,2 \ldots \quad(p-2)
$$

eine Reihe, die, von der Reihefolge der Glieder abgesehen, mit der zweimal wiederholten Folge der quadratischen Nichtreste der Zahl $p$ übereinstimmt. Da nunmehr

$$
g(x) \equiv \prod\left[\left(N_{i}-1\right)\right]^{2} \quad \begin{gathered}
N_{i} \text {-quadrat. Nichtrest } \\
i=1,2 \ldots \frac{p-1}{2}
\end{gathered}
$$

ist. So ist auch

$$
\begin{aligned}
g(x)= & \left\{\left(N_{1} N_{2} N_{3} \ldots\right)-\left(N_{1} N_{2} \ldots N_{\frac{p-1}{2}}-1+\cdots\right)+\right. \\
& \left.+\ldots+\left(N_{1}+N_{2}+N_{3}+\ldots\right)-1\right\}^{2}
\end{aligned}
$$

Nun ist

$$
N_{1} N_{2} N_{3} \ldots \equiv-1
$$


die anderen Summen dagegen (wie leicht aus der Theorie der symmetrischen Funktionen und aus der Beziehung

sobald

$$
\Sigma N_{i}^{s} \equiv 0
$$

$$
\frac{p-1}{2}>s>0
$$

nachzuweisen ist) kongruent 0 sind, somit

$$
g(x)=(-1-1)^{2}=4 .
$$

Nachdem die Berechnung der Werte von $g(x)$ für alle $x$ erledigt ist, gehen wir zur Berechnung der

$$
\sum_{r=1}^{r=p-1} r^{k} g(r)
$$

Jetzt müssen wir dem ind. $y$ bestimmte Werte beilegen. I. Ist ind. $y=(p-1)$, so kommen wir zu dem trivialen Fall, dab $g(x)$ für jedes $x$ gleich 0 ist und $g(x)$ identisch ist $\equiv 0$. Es sei noch erwähnt, daß dieser Fall eigentlich schon frtiher erörtert wurde, mit dem Unterschiede, daß das Produkt früher von $n=1$ bis $n=(p-2)$ jetzt von $n=1$ bis $n=(p-1)$ genommen worden ist, weshalb wir auch zu anderen Resultaten gekommen sind.

II. Ist ind. $y=\frac{p-1}{2}$; so haben wir den Fall, welchen wir eigentlich zu untersuchen haben, da

und

$$
a^{\frac{p-1}{2}} \equiv-1
$$

$$
\left(x^{n}-a^{\frac{p-1}{2}}\right) \equiv\left(x^{n}+1\right) \text {. }
$$

Laut den früher Gesagten

$$
\begin{aligned}
g(x) & \equiv 0, \quad \text { wenn ind. } x \text { - ungerade Zahl, } \\
& \equiv 4, \text { wenn ind. } x \text { - eine gerade Zahl (nicht } p-1), \\
& \equiv 1, \text { wenn ind. } x-(p-1),
\end{aligned}
$$

somit

$$
\sum_{r=1}^{r=p-1} r^{s} g(r)=4\left(a^{2 s}+a^{4 s}+\ldots+a^{(p-3) s}\right)+a^{(p-1) s} 1 .
$$

Fur $s=(p-1)$ oder $\left(\frac{p-1}{2}\right)$

$$
\text { ist } \quad \sum_{r=1}^{r=p-1} r^{s} g(r) \equiv 4 \frac{p-3}{2}+1=-6+1=-5
$$


für alle anderen $s=1+4 \frac{a^{(p-1) s}-a^{2 s}}{a^{2 s}-1}=-4+1=-3$.

Somit ergibt schließlich unsere Zerlegung:

$$
\begin{gathered}
g(x)=5 x^{p-1}+3 x^{p-2}+3 x^{p-3}+\ldots+3 x^{\frac{p-1}{2}+1}+ \\
+5 x^{\frac{p-1}{2}}+3 x^{\frac{p-1}{2}-1}+\ldots+3 x
\end{gathered}
$$

III. Ist weiter ind. $y$ eine andere gerade Zahl, so ist

$$
\sum_{r=1}^{r=p-1} r^{s} g(r)=(-1)^{s}\left(1-y^{2}\right)^{\frac{p-1}{2}}+1 .
$$

IV. Ist schließlich ind. $y$ eine ungerade Zahl und nicht gleich $\left(\frac{p-1}{2}\right)$

$$
\sum r^{s} g(r) \equiv-3+(-1)^{s}\left(1-y^{2}\right)^{\frac{p-1}{2}}
$$

für $s=$ einer geraden Zahl (nicht $p-1)$;

$$
\begin{aligned}
& \equiv-5+(-1)^{s}\left(1-y^{2}\right)^{\frac{p-1}{2}} \\
& \text { fur } s=\frac{p-1}{2} \text { oder } p-1 \\
& =1+(-1)^{s}\left(1-y^{2}\right)^{\frac{p-1}{2}}
\end{aligned}
$$

für $s=$ einer anderen ungeraden Zahl.

Aus der früher durchgefuhrten Zerlegung

$$
\begin{gathered}
\prod_{n=1}^{n=n-1}\left(x^{n}+1\right)=5 x^{n-1}+3 x^{p-2}+\ldots+3 x^{\frac{p-1}{2}+1}+5 x^{\frac{p-1}{2}}+ \\
+3 x^{\frac{y-1}{2}-1}+\ldots+3 x
\end{gathered}
$$

können wir nun schließen, daß die Summe der Anzahlen der Zerfallungen der Zahlen einer arithmetischen Progression

$$
\begin{gathered}
k, k+(p-1), \ldots k+(p-1)\left(\frac{p-1}{2}\right) \\
\text { wenn } \\
k \leqq \frac{p-1}{2}, \\
k, k+(p-1), \ldots k+(p-1)\left(\frac{p-3}{2}\right),
\end{gathered}
$$


wenn

$$
k>\frac{p-1}{2}
$$

entweder $\equiv 5$ ist, wenn $k=\frac{p-1}{2}$ oder $p-1$ ist, $=3$ in allen vibrigen Fällen, wobei wir noch einmal hervorheben wollen $\left(\frac{p-1}{2}\right)=p_{1}$, wo $p$ und $p_{1}$ beide Primzahlen sein mlissen.

Beispiel: $p=11 \quad[A(s)-$ Anzahl der Zerfällungen von $s]$

$$
\begin{gathered}
A(5)+A(15)+A(25)+A(35)+A(45)+A(55)= \\
=3+20+39+31+10+1=104=5
\end{gathered}
$$

Nun dürfen wir auch jetzt einige unsere früheren Schlüsse hier wiederholen:

Summe der Anzahlen der Zerfallungen der Zahlen

$$
k, k+\eta-1 \ldots
$$

in Summen, wo jeder Summand höchstens $s$-mal vorkommen darf, ist $\equiv 3$ oder 5 ;

auch die Surnmen der Darstellungen der Zahlen

in der Form

$$
k, k+1)-1, \ldots
$$

$$
1 x_{1}+2 x_{2} \ldots+(p-1) x_{p-1},
$$

wo

$$
x \leq s
$$

ist $\cong$

3 oder 5. 